\title{
Hard Prison Regime: Is it an Issue of Exclusively Concern to Detainees? An Exploratory Empirical Research into the Experiences of State Correction Officers
}

\author{
Serena G, Giuseppe M and Giuliana La F* \\ Department of Jurisprudence, Libera University Santa Silvia, Roma, Italy \\ *Corresponding author: Giuliana La Fiura, Department of Jurisprudence, Libera University Santa Silvia, Roma, Italy, Tel: 3271786526; E-mail: \\ giulianalafiura92@gmail.com
}

Received date: May 18, 2018; Accepted date: June 22, 2018; Publication date: June 28, 2018

Copyright: ( 2018 Giuliana La F, et al. This is an open-access article distributed under the terms of the Creative Commons Attribution License, which permits unrestricted use, distribution, and reproduction in any medium, provided the original author and source are credited

\begin{abstract}
This study stems from the interest of identifying the presence and influence of the mafia phenomenon in different spheres of everyday life, social and working environment as well. So we tried to analyze the mafia, or rather, the pressure it exerted on the daily context, observing and at the same time trying to focus on its influence over a circumscribed phenomenon of clinical interest such as the burnout found in police officers who have been into contact with prisoners condemned to "hard prison regime". It was used an empirical research method with scientific instruments such as the Maslach Burnout Inventory. The survey of working satisfaction ("Survey of Working Satisfaction", n.d.), and on an ad hoc semi-structured interview. According to the analysis of instruments we didn't find evidence of a direct correlation between the Police Officers job experience and the development of burnout syndromes. On the other, we noticed, on average and with an across-the-board approach, a remarkable presence of anxiety and stress as somatization medically recognized (hypertension arterial, anxious state) and Depersonalization linked to the perceived responsibility.
\end{abstract}

Keywords: Psychology; Prison officers; Mafia; Burnout; Working satisfaction; Empirical research

\section{Introduction}

This study has its deep roots in the studies conducted in the last twenty years on the psychology of mafia phenomenon [1-9] and focuses on the influence that mafia can exert in the onset of the burnout syndrome [10] in the penitentiary police agents. Unlike mafia phenomenon, the psychological-clinical research is determined to study mafia from different point of views: the inner world of "man of honor", the relational and psychopathological structures of its family matrices, the connections between the inner world and the social world, internalized rules and social rules. Moreover, mafia has been able to introduce and impose itself as a totalizing identity within specific and environmental contexts both as a criminal organization and a culture. Mafia phenomenon is based on a rule-based mentality consisting of strict rules and indisputable values at the base of which lays the non-acceptance, the devaluation of the other and of the other than the self. Therefore, people who are within such a value system are forced to take part in this group and accept this culture as the only form of relationship and organization of the self [11-13]. On the one hand, Mafia is able to provide the subject with a psychological sense of power and respect, on the other, to satisfy the needs of narcissistic omnipotence of the individuals who belong to it through the formation of myth of the man of honor $[14,15]$.

As revealed, the central idea of mafia culture is the submission of the other and is accomplished through the creation of a condition of a state of fear by the organization. This pressure is a strategic condition which is fed not so much by explicit violent acts, rather using a specific language of a non-written code embedded in everyone's memory which uses especially the gestures and expressions of the body than the word. Everything is implicit in Mafia language, as in a preverbal communication: everything alludes to something that "is already known" and that it isn't specified. This aspect is perhaps what makes mafia threat even worse or the fact that it is implicit and rarely verbally expressed. Within the mafia clan the individual is overwhelmed by a thought already thought and it is difficult and often terrifying conceive an opposition or even a new idea, [16]; a pathological thought that engulfs and phagocytes the individual who can only accept it or otherwise losing its identity.

According to this premise, it would seem that the "man of honor" himself does not have a subjective identity, because he is unconsciously and entirely conceived by "Cosa Nostra" [16]. This identity relies on family affiliation and social codes that use a one-dimensional vision of reality [17]. This show how exclusively the clan (we are Mafia) is able to explain, give significance to the world and provide identity and security to its members; in fact it saturates the mental space and at the same time deprives the individual of thinking about himself and especially using a different code that isn't shared by the group $[13,17,18]$. Although, mafia has been analyzed in its economic, political, sociological, and historical aspects similarly to any complex phenomenon, we cannot state the same for the attempt to study it through a psychological aspect that is actually quite recent. After psycho-social and psychoanalytic studies a more empirical and systematic scientific approach was inaugurated by group-analysis subjective [19], which, for about fifteen years, has become increasingly more interested in the psychology of the mafia phenomenon trying to center its attention on the psychic aspects related to a certain way of acting and being.

Assumed to be our theoretical model of reading phenomena, the reference theory of subjective group-analysis allows us to analyze cultural and behavioral codes of Cosa Nostra because takes into 
consideration the specificity of the social, anthropological, cultural context of reference. We can state that this complex phenomenon profoundly influences both the territory where people's culture and beliefs are oppressed, and people who strongly refuse it. In fact, once we took in consideration the prison context where penitentiary agents work; we noticed that mafia's value system exerts influence on those workers who establish contacts with prisoners. The Penitentiary agents we examined belong to a special group of the Penitentiary Police Corps named "Gruppo Operativo Mobile" with intent to closely monitor and control detainees under the "hard prison regime" [20]. Hard prison regime involves the suspension of ordinary prison sanction rules and supplementary strict limitations on the prisoner's freedom more severe than those applied to common detainees; this suspension is applied to certain cases contemplated by Art. 41-bis of the Law of 26 July $1975 \mathrm{n}$. 354 in the field of "Penalty Settlement Standards") [21]. Specifically, this study centers on criminals who committed crimes related to the organized criminal activity. The aim of this research is therefore to investigate giving an analytic look to the relationship between agents and prisoners, and demonstrate how it can produce a psycho-physical reaction caused for work. As claimed, in fact, by some authors, and shown by various researchers, there are some jobs such as the Penitentiary Agent that can cause to workers more severe psychological effects, work stress syndromes and burnout [22] than other types of jobs.

The term "burnout" refers to all disorders to which workers are undergone which arise from the overwhelming demands required by work environment [23]. Firstly, a progressive alienation, an emotional divestment with simultaneous physical symptoms represent a clear signal for these disturbances and may include a wide range of diseases like sleep disorders and gastrointestinal diseases. Secondly, behavioral problems can range from impatience and impulsiveness to relationship problems while cognitive-affective problems can range from an initial emotional detachment to true cynicism. In conclusion, this study with its exploratory approach brings an innovative and transverse modality useful to analyze both the phenomenon of mafia and work-related stress syndromes, and allows us to provide new elements of knowledge and observation. In addition, the use of the theory of complexity allows us to observe a phenomenon so articulated from many point of views. Like a cubist painting, we established a correlation between the phenomenon and all that directly and indirectly is related to it giving substance to new questions and connections not investigated by individual disciplines. In conclusion, we have adopted this overall view to analyze this phenomenon. The complexity of mafia phenomenon, the complexity of its development, its ability to spread across society and to survive has been the premises to study the ways in which reveals itself. We didn't underestimate potential secondary or primary elements in the development of other issues, such as the psychological ones within the work place. It's necessary to study the connection between several phenomena (in our case burnout and mafia) and the modality this bond is structured.

\section{Research Methodology}

This study was carried out by both the administration of two clinical diagnostic instruments (MBI, Maslach Burnout Inventory; QLS, Work Satisfaction Questionnaire) and an ad hoc semi-structured interview consisting of about 45 questions, using the case study method [24] which is characterized by an exploratory type of research. Firstly, these questions are supposed to examine the emotional response deeply felt by the subjects of our research at working as prison officers, in the field of "Penitentiary System Settlements"; "Penitentiary Police Department", n.d.), secondly, how work affects their private life, and last but not least how mafia and living in close contact with it, inside and outside prison, affects their lives, choices and relationships. Upon an informal agreement, four male penitentiary agents, all of them in their fifties, accepted freely to be involved in our research. Although, an exploratory study was considered as appropriate considering the specific factors that qualify our "subject", it cannot be considered valid because the statistics survey isn't remarkable and does not allow us to extend and generalize the results. On the contrary, number processing allows a careful qualitative assessment able to suggest new and deeper questions to this study. The formal and material complexity of the material as well as its subjective dimensions demanded for a quality research model which refers to the epistemological paradigm of complexity [25].

Currently, the scientific literature spurs researchers who work in this research area to use qualitative instruments able to handle a wide range of such variables. The Grounded Theory [26] can be considered a clear example of this instrument as it consists of an accurate collecting data through journals, interviews, focus groups etc. and it structures them in categories. After taking into account the analysis of the data, we found out each small-scale individual value of the MBI and identified the Low, Medium and High value of each subject. The results of the different subjects to the MBI were then compared identifying the percentage frequencies of the different small-scale levels; finally the Average and Standard deviations of the results detected were obtained from the test of the MBI through a comparison with the regulatory sample for the Italian version of the instrument. Regarding the QLS [27] (Work Satisfaction Questionnaire, n.d.) several degrees of General Satisfaction have been detected in the four subjects examined; standard averages and standard deviations of these subjects were compared with the Standard and Standard Deviations of the normative sample [28]. For the analysis and processing of the interview was adopted the inductive method with reference to the Grounded Theory [26]. The three observers were interviewed separately and after analyzing and comparing the results achieved we identified and chose the most suitable categories of analysis to segment the collected data.

Later, we proceeded to extract the main issues useful to draw the thematic-conceptual figures. The quantitative and qualitative data collected were read through a global comparison which allowed us to get a detailed view on the individual and a wide view on the entire sample. If we consider this small number of individuals taken into consideration, this study assumes that new hypothesis can be supported concerning the relationship between mafia phenomenon and the onset of Burnout's syndromes in prison officers. The study of these four cases allowed us to formulate new hypothesis that must be undergone to a following wide-scale test.

\section{Analysis and Data Processing}

Firstly, we identified each small-scale value of the MBI $[27,28]$ and highlighted the Low, Medium, or High value of each small-scale for each subject. A second point was the comparison of the results of each subject of the MBI and the identification of the percent frequencies of the levels of the different small-scales. It was found a low level of burnout in subjects to whom the test was given. Moreover, low levels of Emotional Exhaustion and Depersonalization were found in a higher percentage and average percent levels of Personal Fulfillment (3 out of 4). 
Citation: Serena G, Giuseppe M, Giuliana La F (2018) Hard Prison Regime: Is it an Issue of Exclusively Concern to Detainees? An Exploratory Empirical Research into the Experiences of State Correction Officers. J Foren Psy 3: 139. doi:10.4172/2475-319X.1000139

Page 3 of 8

In addition, the Average and Standard Deviations obtained from the results of the MBI test were later compared with the normative sample for the Italian version. In relation to the Average referring to the subscale Emotional Exhaustion was revealed a lower score than the subscale Personal Realization that was clearly larger. Finally, there is a markedly higher score relating to the sub-scale Depersonalization (Tables 1 and 2).

\begin{tabular}{|l|l|l|}
\hline \multicolumn{2}{|l|}{ Subjects of the research $(\mathbf{n}=\mathbf{4})$} \\
\hline & Ma & Dev.S \\
\hline EE & 17,50 & 10,34 \\
\hline Dp & 12,75 & 8,01 \\
\hline RP & 32,25 & 4,11 \\
\hline
\end{tabular}

$\mathrm{EE}=$ Emotional exhaustion, $\mathrm{Dp}=$ Depersonalization, RP=Personal realization, $\mathrm{Ma}=$ Average, Dev. $S=$ Standard deviation

Table 1: Subject of the research.

\begin{tabular}{|l|l|l|}
\hline \multicolumn{3}{|l|}{ Regulatory Sample (n=1779) } \\
\hline & Ma & Dev.S \\
\hline EE & 19,47 & 11,33 \\
\hline Dp & 5,34 & 5,44 \\
\hline RP & 33,99 & 8,28 \\
\hline $\begin{array}{l}\text { EE=Emotional } \\
\text { Ma=Average; Dev.S=Standard deviation }\end{array}$ \\
\hline
\end{tabular}

Table 2: Regulatory sample.

According to the QLS (Work Satisfaction Questionnaire, n.d.) the four subjects examined revealed several degrees of General Satisfaction. Once the QLS were submitted to each subject the analysis show a wide divergence of responses in some subdivisions because they highlight a greater presence of low scores both in the small-scales that indicate satisfaction with the salary, and the professional/ developmental enrichment and with managers/superiors. On the other, we noticed an almost unanimous presence of high scores in the subdivisions which refer to satisfaction with engagement and task. Additionally, high scores were also detected with reference to satisfaction with communication whereas the other subdivisions vary very slightly as compared to the normative sample with no peaks to be analyzed systematically on a timely manner. In conclusion, there is also evidence of a slightly high score in the General Satisfaction index of the subjects examined compared to the normative sample (Tables 3 and 4).

\begin{tabular}{|l|l|l|}
\hline Scale & Avergage & Ds \\
\hline Salary & 16.00 & 4.76 \\
\hline Professional Enrichment & 10.00 & 3.56 \\
\hline Managers /Bosses /Leaders & 13.25 & 4.19 \\
\hline Participation & 19.50 & 2.65 \\
\hline Recognition & 15.50 & 3.11 \\
\hline
\end{tabular}

\begin{tabular}{|l|l|l|}
\hline Working conditions & 15.75 & 3.4 \\
\hline Relationships between colleagues & 16.25 & 2.99 \\
\hline Role & 18.00 & 3.37 \\
\hline Communication & 17.00 & 2.94 \\
\hline Overall satisfaction Index & 141.25 & 7.63 \\
\hline
\end{tabular}

Table 3: Average and standard deviation of the sample examined $(\mathrm{N}=4)$.

\begin{tabular}{|l|l|l|}
\hline Scale & Media & Ds \\
\hline Salary & 11.76 & 4.24 \\
\hline Professional Enrichment & 15.09 & 4.20 \\
\hline Managers /Bosses /Leaders & 18.93 & 3.69 \\
\hline Participation & 21.02 & 4.41 \\
\hline Recognition & 15.23 & 4.32 \\
\hline Working conditions & 13.85 & 3.36 \\
\hline Relationships between colleagues & 17.83 & 3.45 \\
\hline Role & 17.33 & 3.37 \\
\hline Communication & 12.24 & 2.98 \\
\hline IOverall satisfaction Index & 139.00 .00 & 22.32 \\
\hline
\end{tabular}

Table 4: Average and standard deviation of the normative sample $(\mathrm{N}=3.439)$.

\section{Thematic-Conceptual Maps}

As mentioned above, for the analysis and processing of the interviews it was adopted the inductive method with reference to the Grounded Theory [26]. After an informal observation of the phenomenon we have pinpointed the most suitable units to segment it or categories.

The analysis of data follows a "coding process" which allows us to identify conceptual categories in the collected data. Being an empirical research, the selection of the categories of analysis to establish the most suitable categories to segment the material was done by making a comparison between the three observers who analyzed separately the interviews given to the four subjects. Thanks to this methodological process we selected the main issues. The analysis of the thematicconceptual areas below, has the purpose to explicate, clarify and show the relevant results obtained from the interviews. As revealed, they don't take in consideration the unlimited complexity that arises from communicative and relational twines, but it's a synthetic attempt of scientific sharing of something that would be difficult and tedious to disclose (Figure 1). 


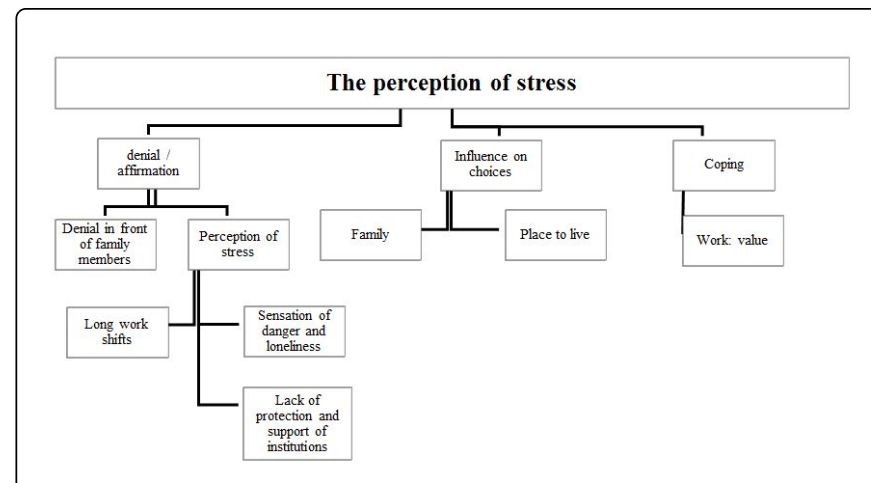

Figure 1: The perception of stress.

The first thematic area extrapolated from the comparison of the interviews is the area related to the stress perception in the specific field of work of Penitentiary Police Corps. Firstly, we noticed the agents revealed an inability to communicate their own moods because they don't want to appear weak (in $80 \%$ of the participants). Subsequently, their aim is to protect their family peace because it is considered as the only "safe haven" where to take refugee and unplug from work at the end of the day. The motivation that guides these actions seems to be "to protect" which also involves the acceptance of stress and the implementation of copying strategies intended to negate, or keep their distance from the possibility of eliminating stress changing job, because we live and work in a place where there are a few job opportunities (in the South of Italy), and having a good job is considered a lifelong ambition.

Consequently, the choice to work in a jail it seemed to them the only opportunity in that moment of their lives to achieve their own independence from the family of origin (in $90 \%$ of the participants). As a consequence, they had to accept to maintain "the permanent job" with a suitable fee even knowing that the result would have been losing the privilege to astonish, be afraid, and last but not least to experience strong emotions. They produce strategies of copying focused on making the ties between private life and job stronger. Although the subjects have been trying to put distance between job and private life, they state that they have been influenced by their work in life choices. Moreover, it comes to light a strong sense of solitude which hides a need for emotional support and understanding.

According to this, the only emotional support seems to be the group of colleagues who show off a strong sense of belonging (in $80 \%$ of the participants). This group becomes a source of emotional resources from which the subject draws energy to cope with complex situations and often not easily sustained psychologically (such as fights, suicides or attempted murders). It was also revealed a strong feeling of abandonment by the authorities of the State (in 70\% of the participants). In fact, when they speak, the word "protection" is frequently used both related to their family environment which must be kept away from the hard working reality, and refereed to themselves especially in relation to those who should be members of this function or the members of the State.

It is clear that these dearth's create fertile ground for the arise of stress related to work that as time goes by it turns into a complete flaw of interest for their own work. Carrying out this work it is necessary to maintain some emotional detachment precisely because often the emphatic demands of the detainees are greater than an agent is prepared to face. This detachment, although defensive and necessary, it can increase excessively causing burnout: this probably due to the lack of an appropriate psychological support. A considerable distance, dialogue and awareness of their roles are essential elements in creating balanced relationships and not worn out among prison officers and detainees. According to what has been said, burnout syndrome $[1,10,22]$ appears to be present but denied by the subjects themselves (in $85 \%$ of the participants). It is faced in solitude, with a few resources available, showing physical and psychic problems which show its presence in the form of hypertension, anxious states and irascibility (Figure 2).

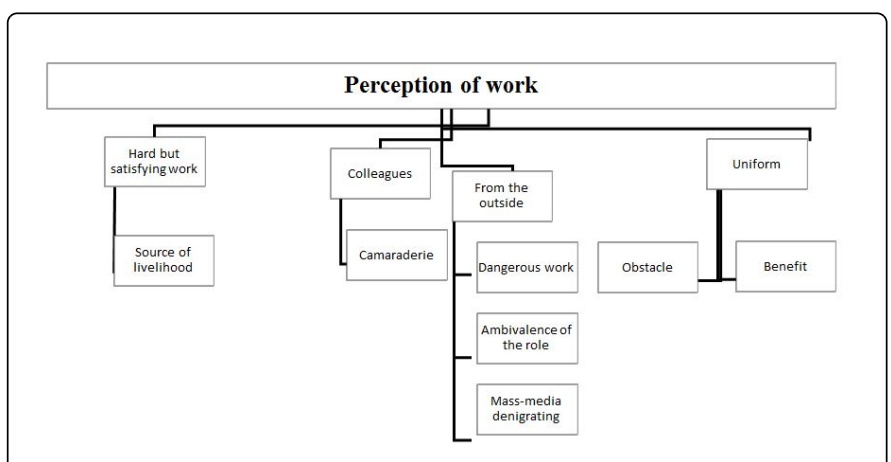

Figure 2: Penitentiary police: What job difficulties?

A new area stems from the first thematic area which allows us to understand in a clearer way how the agents perceive their work and as they believe it is perceived by other people on the basis of the comparison made with the feedback received. The subjects, representing themselves as agents, try to keep an image of themselves as professional and qualified workers emphasizing their ability to maintain close ties between them and the detainees, highlighting their capacity for non-judgment of the prisoners. They complain of not being fully understood because they are considered simply "Prison guards" (definition perceived as denigrating), like people who only open and close the cell (75\% of the participants). Someone (30\% of the participants) also refers that the mass-media only report beatings to detainees or corruption of some agents without ever talking about issues that need to be tackled every day and the risks that this craft incurs.

Making a group with colleagues, that we call "camaraderie", is a strategy of copying seen as a form of solidarity to support colleagues. In the perception of their work they often identify themselves with the "uniform" and with what it represents as a guarantee which allows them to keep the net boundaries in the execution of their work between themselves and the detainees. On the contrary, the cultural and social sphere is lived in another way even though we must report that the opinions are not unanimous in all prison guards interviewed. Inside and outside the subjects emerges an ambivalent vision and a clear separation, between the working self and the self which comes back home (in $85 \%$ of the participants). It is a kind of doubling that in some cases is not so obvious, because it turns into physical and psychic syndromes that have negative effects on personal life. It seems that the Penitentiary Police Agent puts in place a process that implies a constant search and affirmation of net boundaries, both between a psychic inside and an outside (inside and outside the uniform), and a physical one (inside, during and out of work, in their personal life). An attempt like this might draw the fear which will lead them into a 
process of Depersonalization (which is one of the sub-scales found in the studies on burnout syndrome).

If we take into consideration the definition of boundaries, it is necessary to underline that within a detention institution they create a community within it, a contest with its own rules, rhythms, lifestyles and work, in which coexist relationships between those who live there and those who work there several hours per day. Detainees are not the only ones who live and observe the rules within this community, even the Penitentiary Agents are involved in this sort of similar life, and obviously the significant difference is that agents leave the prison walls at the end of their work. The relationships between detainees and agents as a consequence are ambivalent and characterized both by the respect of the detainees as human beings, and from the dependence detainees have on agents, so the former establish stable relationships and meanwhile they require the maintenance of a certain distance. Taking the distance and the direct emotional involvement into consideration, this ambivalence is amplified by the fact that agents live in close contact with detainees every day, and often become the only one to whom detainees can speak and pour out their emotions.

In light of this close involvement, the human contact between the agent and the person who committed the crime is inevitable and the only symbol that emphasizes the asymmetry of this relation turns out to be the uniform. The tasks required and, which are obviously not simple targets to reach are Keeping the Order, Respecting the Rules, Respecting the Prisoners, Humanizing the relationship between agents and detainees themselves (Figure 3).

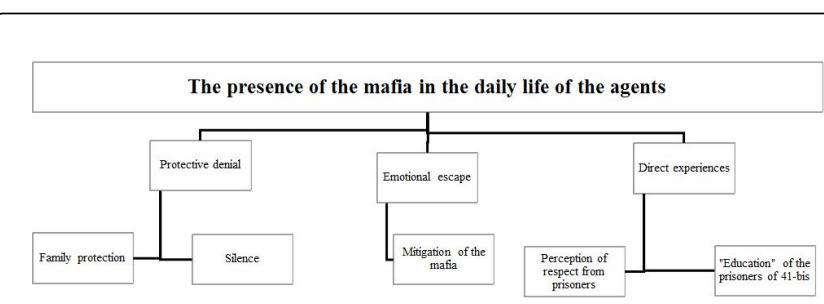

Figure 3: The presence of the mafia in the daily life of the agents.

As we have seen the relationship between the mafia phenomenon and the agents' daily life is a cross-cutting thematic area, because the presence of the mafia phenomenon casts a dark shadow over the daily and working lives of the interviewed agents. The presence of the mafia is perceived and approached through coping strategies that push subjects towards a detachment and an "emotional escape" over the phenomenon. For instance, they don't speak with their family members to preserve the safe shelter represented by home and family (in $75 \%$ of cases). The attempt to dissociate work from private life keeps recurring: they hold back their thoughts avoiding to stress and scare the private and safe contest of their families. The "emotional escape" is the instinctive reaction to the weak attempt to deal with the possibility of addressing the "Mafia" subject. It is expressed through a description of the phenomenon which uses common and shared definitions which have the power to place a barrier and neutralize the negative effect of the phenomenon itself.

The emotions originate from this phenomenon are denied to make the attempt to maintain a suitable detachment from it, guarantee a psychic serenity and draw clear boundaries between the perception of the self as agent and the internal representation of the detainee. The mafia phenomenon is thus merged into the background and perceived as a constant but veiled presence of working life and daily life. The fear toward its ability to contaminate every aspect of life and everything which keeps in contact with it, creates the need to limit this phenomenon to recognizable spaces and therefore easily avoided. On the other hand, the application of the "Hard Prison Regime" against those who committed mafia crime offenses favors the reduction or sometimes the loss of their power and as a consequence of their monolithic identity thanks to the impossibility of the subject to maintain the relationships with the criminal organization which will "leave them out".

According to the perception of the agent, mafia detainees are controllable because, being detainees; they are exclusively present in the working contest, far away from home and their serenity (according to $95 \%$ of the participants). Mafia is a reality that can be psychically represented on a gelstalt background. The agents try to merge it into this background, because it seems to be remote and controllable at a safe distance, an emotional distance which operates as a protective armor to preserve the boundaries which lay at the base for maintaining their own identity (Figure 4).

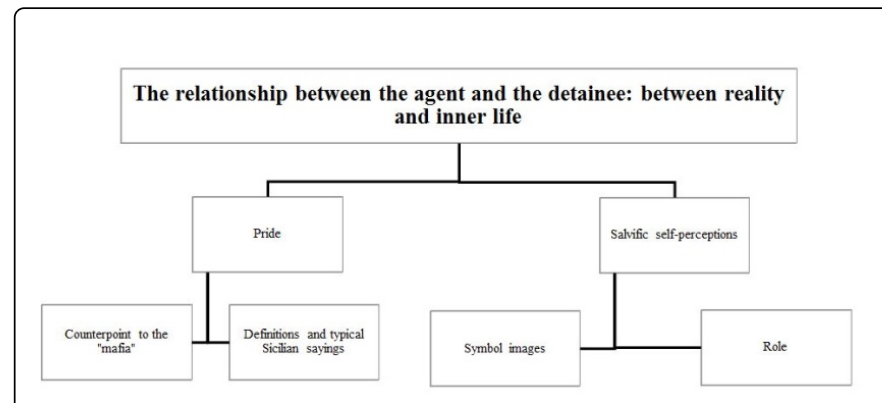

Figure 4: The relationship between the agent and the detainee: between reality and inner life.

Relating to this conceptual-thematic area, the identity of the agents is protected also thanks to the perception that they have of themselves and their role. Penitentiary Police exercises its power taking on the responsibility that everything must be done in compliance with the rules. Stressful situations like lies among prisoners, allusive threats, and rebellions can be difficult to handle with. Its role is clearly defined by the juxtaposition with the detainees or those people who didn't respect the law. It can be difficult to distinguish their personal opinions from their professional duties, to control emotions as anger and fear and do not let that what they live between the prison walls affects their cultural and social life outside.

Used as a defensive way, pride is mainly manifested in the salvific and control imagines that they choose to describe (in $75 \%$ of the participants). If we consider the way the subjects analyzed perceive their task, pride is intensely showed off because it plays a key role: in fact, the agent might consider its role useful for the maintenance of the public security; even though it is a stressful job it is relevant to the community. This manifestation of pride is in contrast with the way the mass-media report and also with what the ordinary people consider the Penitentiary Police's work is.

The negative emotions such as the frustration at not being considered by the institutions and misunderstood by the outside world within the prison environment, are often balanced by the feeling of pride fueled by the exaltation of its social and public importance of their role (in $60 \%$ of the participants). They are pride of being a 
policeman and not a "sbirro", which is the pejorative word used by men of mafia to mean a person who does not stay on their side. The mere fact of not being part of their world, the individual is without value, is "nuddu mmiscatu cu nienti" (no-one mingled with anything) which is the heaviest attempt to annihilate their function. Claiming the importance of their role, the agents try to overthrow the contemptuous judgment on them by emphasizing their image and identity as indicated in the subjective Group-analytical perspective $[19,29,30]$. Both the penitentiary institutes, and the agents and mafiosi create a fertile ground to the psychological-clinical research that, in this context intends to investigate the type of relationships and interactions which arise and intersect the cultural, social and transpersonal aspects of the social actors described.

\section{Results}

If we compare the data emerged from the administration of the tests (QLS e MBI) [27,28] and the semi-structured interview ad hoc planned, is possible to trigger a critical way of thinking on the goals of this research generating further study hypothesis. The analysis of this sample allowed us to observe the mafioso phenomenon from a different perspective in other words that of workers. We immediately noticed that during the performance of their duty they are exposed to the mafia world in which everything has a precise position, even the "sbirro". This display exerts influence not only in the workplace of the subjects we mentioned, but also in their privacy and in the way they perceive their work. What we have said can give useful directions for potential research relating to this topic.

As far as the results of this research concerned, concerning the subdimension of involvement it was detected a low level of burnout, a high grade of general satisfaction and a relative satisfaction. On the contrary, low levels of work satisfaction have been detected related to some aspects such as the presence of salary which is not adequate to the duties and timetables, after poor levels of satisfaction even with regard to the possibilities of enrichment or career in part attributed to a lack of attention by superiors. Moreover, interviews show a high work-related stress [31], and a high degree of frustration in relation to how their work is considered by people outside the prison. The frustration is generally expressed with feelings of dissatisfaction, anger and sometimes even with an ostentation mischief. However, we have also noticed mistrust and paranoid feelings towards the outside, evident by the difficulty encountered requiring their consent to be undergone the study. We obtained their accession once we accepted their repeated request for anonymity.

Mistrust and paranoia sometimes turn into emotional detachment. If we analyze their words, Mafia is described only through common terms and mythical representations, on the contrary only its violent manifestations are criticized and ignored. This means that the fear of becoming a victim of mafia has actually a great influence on the subjects we examined beyond the "saving" construction to justify its role. Anxiety in having to handle a job with dangerous subjects, even if confined to the prison, is therefore the basis for the emotional posting useful to handle the stress of their work. On the one hand, the instruments we used did not detect significant data of syndrome of burnout, $[28,30]$, on the other, the presence of anxious states and in some cases of hypertension could witness a high rate of stress. Burnout syndrome is detectable in a medium value and seems to be directly correlated with variables such as salary, relationships with superiors, anxiety created by responsibilities, the shaky work shifts, the lack of protection and support from the institutions of government. However, it is possible to detect indirectly a remarkable anxiety which emerges from the responsibility of having to control and guarding persons involved in Mafia crimes only after a careful observation.

This study of cases applied on four agents, allows us to broaden our observations and the implications that the mafia phenomenon can create, affecting indirectly, even in a hidden way, the perception of their work as generally anxious and stressful. The overall target of the research is partly demonstrated by indicating an indirect influence between the mafia phenomenon and agents' lives especially with regard to the choice of coping strategies more effective in trying to exorcise the fears associated with the Mafia presence on the territory. Otherwise, the specific goals of finding connections between burnout and the phenomenon mafia, as well as detecting the presence of burnout, are dismissed showing no direct influence.

\section{Conclusions}

This study has allowed a wide-ranging observation which used the theory of complexity [28] and the Subjective Gruppanalitica perspective to explore, [21,31], efficient to carefully observe how the Mafia phenomenon enters lives and cultures in an indirect way, a hidden away, hitting seriously its victims, concealing its danger mingling with everyday life.

Studying the theories of psychology of the mafia phenomenon $[29,32,33]$, in relation to its codes, and on its way to penetrate and "parasitize" the territory where it acts [5] we made the attempt to understand what are the bases of the mafia way of thinking.

This research, starting from the exploratory case study method allows us to look at the mafia phenomenon, its presence in the daily contest starting from the experience of the penitentiary agents, both inside and outside the prison, and then paving the way for an observation potentially full of ideas for a future consideration. This method appears to be valiant for the study of the dynamics that exist between mafia-style inmates and prison police officers and also it may favor the development of work-related stress syndromes allowing us to bring new elements of knowledge and observation. However, this study is limited by the exiguous amount of cases-it is a sample due to the difficulty to look for available subjects to whom undergo a type of research which puts a person in front of their archaic fears and the possibility to struggle with their own ability to distinguish legality from illegality, the self-perception and the external feedback, the identity and the non-identity $[8,13,16]$. The critical thinking is stimulated by the attempt to understand what the direct impact of mafia is, its codes and its ability such as a chameleon to change and adapt to different contests.

The presence of mafia can also be detected in a stressful work environment through a direct observation, such as penal institutions can represent (analyzed in this study) in which the responsibility perceived in having to keep mafia prisoners create an anxiety particularly relevant and active process of denial and "emotional escape". This 'escape' turns into mistrust, fear of retaliation, inability to freely communicate their emotional status [34,35] and expresses itself also through resistances and wastes that testify the need of protection and legal safety by authorities of government.

New important factors have come to light from this study, as revealed penitentiary agents have difficulty with defining useful boundaries for the protection of their identity and emotional wellness. Furthermore, a limit is represented both by the shortage of diagnostic 
tools useful to thoroughly analyze the phenomena observed and the use of an interview, not standardized and repeatable in results, built ad hoc. Moreover, the instruments have been modified and adapted to the phenomenon to be investigated on the basis of the areas of interest designed for the identification of the research goals; we evaluated some variations taking into account the type of study to be carried out (empirical-exploratory), the number (not known a priori) of the subjects to be submitted to the study and the difficulty of analyzing a complex phenomenon.

Overall, the general aim of this research was to create an opportunity to think on the capacity of the mafia phenomenon has to penetrate into the most diverse contests, reaping victims not only with direct violence (massacres, murders) exercised on people who oppose this phenomenon, but also infiltrating in everyday life and in the common thought of people: mafia represents an all-pervading and silent presence that fills the emptiness that civil culture and institutions cannot fulfill. Mafia is a phenomenon which has learned how to adapt, survive and hide. Challenging this phenomenon we need to know it, explore the psychic dynamics that created it and support it.

Ask new questions and explore new areas of mafia penetration can be a successful way to examine mafia in depth to open up to new reflections, not forgetting to proceed by doing "Science with conscience". The reflection on the mafia must remain open to new ideas that can be useful to understand the trans-psychic connections that form the basis, creating from these assessments a critical thinking, and a new observation point on the phenomenon.

\section{Compliance with Ethical Standards}

\section{Informed consent}

Standard procedures for gaining informed consent were used, and participants were informed of their right to withdraw from the study at any point without having any obligation to explain their reasons for withdrawing.

\section{References}

1. Maslach C, Jackson SE, Leiter MP (1997) Maslach burnout inventory. Evaluating stress: A book of resources 3: 191-218.

2. Giunta S, Lo Verso G, Mannino G (2013) The mafia world: between clinical practice and interventions in the polis, CSR, Genova.

3. Lo Verso GL, Coco GL (2003) The mafia psyche: Stories of clinical cases and collaborators of justice. Milano: FrancoAngeli, Italy.

4. Caleca A (1998) The Mafia inside: Psychology and psychopathology of fundamentalism. Milano: FrancoAngeli Publications, Italy.

5. Lo Verso G, Lo Coco G, Mistretta S, Zizzo G (1999). How the mafia changes. Judicial and psychotherapeutic experiences in a changing country. Milano: Franco Angeli Publications, Italy.

6. Lo Verso G, Coppola E, Giorgi A, Giunta S (2013) The experiences of psychotherapists who work with patients belonging to the mafia world. Further study of the mafia psyche, in Lo Verso G, Mafia and psychotherapy. Milano: Franco Angeli Publications, Italy.

7. Schimmenti A, Giunta S, Lo Verso G (2014). Mafia women: A study of language and mental representations of women engaged with mafia members. IJCS 3:267-274.

8. Giunta S, Mannino G, Lo Verso G (2017). The betrayed Dignity. A psycho-social study on the crime of white-collar workers. Milano: Franco Angeli.
9. Giunta S, Mannino G, Bizzarri C, La Fiura G (2018). Being Mafia Children: An Empirical Transgenerational Research. MJSS. ISSN 2039-2117. Doi: 10.2478/mjss-2018-0018 .

10. Maslach C, Schaufeli WB,Leiter MP (2001). Job burnout. Annual review of psychology, 52:397-422.

11. Coppola E, Giorgi A, Giunta S, Lo Verso G (2009) Territories in backlight, psychological research on mafia phenomenon. Milano: FrancoAngeli Publications, Italy.

12. De Rosa C, Giunta S (2010) Mafias and psychological underdevelopment. In Ravveduto. Roma: Aliberti.

13. Gullo S, Lo Coco G, Di Fratello C, Giannone F, Mannino G, Gary Burlingame, (2015), "Group climate, cohesion and curative climate. A study on the common factors in group process and their relation with members attachment dimensions", in Research in Psychotherapy: Psychopathology, Process and Outcome

14. Falcone G (1992) I, Falcone, I'll explain what is the mafia. In: Unity, 31.05.1992

15. Mannino G, Giunta S (2015) Psychodynamics of the Mafia Phenomenon: Psychological-Clinical Research on Environmental Tapping and WhiteCollar Crime. World Futures: J New Paradigm Res 71: 185-201.

16. Giunta S and Campo R (2005). Identity and fundamentalist cultures. Telling the group. Clinical and social perspectives, 3:34-48.

17. Giunta S (2012). A per-course through the psychology of the mafia phenomenon, -IJPE 4: 139-148).

18. Giunta S, Lo Verso G (2012). In the name of the Godfather, IJPE 4 : 90-107.

19. Lo Verso G, Di Blasi M (2012). Subject Group Analysis. Milano: Raffaello Cortina Editore.

20. GOM Gruppo Operativo Mobile. (n.d.) Disponibile da

21. Legge 26 luglio 1975 , n. 354 .On the subject of: Norms on the Penitentiary Law and on the Execution of the Private and Limitative Measures of Freedom

22. Cherniss C (1980) Staff burnout: Job stress in the human services . Beverly Hills, CA: Sage Publications.

23. Antonovsky A (1987). Unraveling the mystery of health: How people manage stress and stay well CJA.7:77-79.

24. Eisenhardt KM (1989) Building theories from case study research. Academy of management review, 14:532-550.

25. Morin E (1986). Science with conscience. Milano: FrancoAngeli Publications, Italy.

26. Glaser B, Strauss A (1967).The Discovery of Grounded Theory: Strategies for Qualitative Research, Chicago, Aldine Publishing Company.

27. Work satisfaction questionnaire. (n.d.). In occupational psychology. Online, Psyjob.It.

28. Tiraboschi M,Fantini L (2009) The single text of health and safety at work after the correction. (D. Lgs. n. 106/2009) (No. 81-2008). Giuffrè Editore

29. Giunta S, Lo Verso G. (2011). The Mafia, The Mind, The Relationship: Analytical Group- Subjective Studies, Quaderno Report, Csr Coirag, N. 15.

30. Giorgi A, Giunta S, Coppola E and Lo Verso G. (2009). Territories in backlight. Psychological research on the mafia phenomenon. Milano: FrancoAngeli Publications, Italy.

31. Bakker AB, Demerouti E and Sanz-Vergel AI. (2014). Burnout and work engagement: The JD-R approach. Annu. Rev. Organ. Psychol. Organ. Behav 1:389-411.

32. Mannino G, Giunta S, Buccafusca S, Cannizzaro G, Lo Verso G (2015) "Communication Strategies in Cosa Nostra: An Empirical Research" World Futures: J New Paradigm Res 71:153-172.

33. Mannino, G, Giunta S and La Fiura G. Psychodynamics of the Sexual Assistance for Individuals with Disability G. Sex Disabil 35:495-506.

34. Mannino G (2013) Soul, Culture, Psyche. Generative relationships, Milan, Milano: FrancoAngeli Publications, Italy.

35. Mannino G, Caronia V (2017) Time, well-being, and happiness: A preliminary explorative study. J New Paradigm Res 73(4-5):318-333. 
Citation: Serena G, Giuseppe M, Giuliana La F (2018) Hard Prison Regime: Is it an Issue of Exclusively Concern to Detainees? An Exploratory Empirical Research into the Experiences of State Correction Officers. J Foren Psy 3: 139. doi:10.4172/2475-319X.1000139 